Research Paper

\title{
Optimization of Optical Excitation of Upconversion Nanoparticles for Rapid Microscopy and Deeper Tissue Imaging with Higher Quantum Yield
}

\author{
Qiuqiang Zhan ${ }^{1,2}$, Sailing He1,2,3, ${ }^{凶}$, Jun Qian², Hao Cheng², Fuhong Cai ${ }^{2}$ \\ 1. ZJU-SCNU Joint Research Center of Photonics, Centre for Optical and Electromagnetic Research, South China Academy of Advanced \\ Optoelectronics, South China Normal University (SCNU), 510006 Guangzhou, P. R. China. \\ 2. Centre for Optical and Electromagnetic Research, JORCEP, Zhejiang Provincial Key Laboratory for Sensing Technologies, Zijingang \\ campus, Zhejiang University (ZJU), 310058 Hangzhou, P. R. China. \\ 3. Department of Electromagnetic Engineering, Royal Institute of Technology, 10044 Stockholm, Sweden.
}

$凶$ Corresponding author: sailing@jorcep.org.

() Ivyspring International Publisher. This is an open-access article distributed under the terms of the Creative Commons License (http://creativecommons.org/ licenses/by-nc-nd/3.0/). Reproduction is permitted for personal, noncommercial use, provided that the article is in whole, unmodified, and properly cited.

Received: 2013.02.01; Accepted: 2013.02.27; Published: 2013.03.23

\begin{abstract}
Relatively low quantum yield (QY), time-consuming scanning and strong absorption of light in tissue are some of the issues present in the development of upconversion nanoparticles (UCNPs) for biomedical applications. In this paper we systematically optimize several aspects of optical excitation of UCNPs to improve their applicability in bioimaging and biotherapy. A novel multi-photon evanescent wave (EW) excitation modality is proposed for UCNP-based microscopy. The scanning-free, ultrahigh contrast and high spatiotemporal resolution method could simultaneously track a few particles in a large area with a speed of up to 350 frames per second. The HeLa cancer cell membrane imaging was successfully performed using $\mathrm{NaYF}_{4}: 20 \% \mathrm{Yb}^{3+} / 2 \% \mathrm{Er}^{3+}$ targeting nanoparticles. Studies with different tissues were made to illustrate the impact of optical property parameters on the deep imaging ability of $920-\mathrm{nm}$ band excitation. In the experiments a semiconductor laser with a $920 \mathrm{~nm}$ wavelength was used to excite UCNPs in tissue phantom at five depths. Our experimental and computational results have shown that in UCNP-based diffusion optical imaging with 920-nm laser excitation could lead to larger imaging depth range compared to traditional 974-nm excitation in a wide dynamic range of tissue species. As the QY is power density dependent, a pulsed laser is proposed to improve the QY of UCNPs. This proposal is promising in drastically increasing the imaging depth and efficiency of photodynamic therapy.
\end{abstract}

Key words: upconverting, total internal reflection, pulsed excitation, deep imaging, quantum yield.

\section{Introduction}

Upconverting nanoparticles (UCNPs) constitute a novel type of contrast agent with interesting and unique properties for luminescence bio-imaging1. Superior to organic dyes and quantum dots (QDs), UCNPs enable autofluoresence-free imaging under near-infrared excitation ${ }^{2}$. The research topic of UCNPS has become popular for biomedical applications, particularly optical bioimaging. UCNPs exhibit practi- cally no autofluorescence, no photobleaching, no blinking, large anti-Stokes shifts, sharp emission band, deep detection ability and a high spatial resolution ${ }^{3,4}$. In recent years, UCNPs have therefore attracted much attention in the biophotonics area. They have widely been employed in in vitro microscopy ${ }^{5-7}$, small animal imaging 8,9 , diffusion optical tomography ${ }^{10}$, multimodal animal imaging11, 12, high sensitivity biosens- 
ing $^{13,14}$ and photodynamic therapy (PDT) ${ }^{15,16}$.

However, there are still some challenges (despite the unique optical property and rapid development of this nanoparticle), such as relatively low QY17, 18, time consuming scanning 19,20 and strong absorption of excitation light in issue ${ }^{21-23}$. Although fluoride-based inorganic upconverting nanoparticles of $\mathrm{NaYF}_{4}: \mathrm{Er}^{3+} / \mathrm{Yb}^{3+}$ are significantly more efficient for multiphoton excited fluorescence when compared to quantum dots, gold nanoparticles and organic dyes ${ }^{18}$, the absolute QY is still on the magnitude order of $1 \%$ (absolute value depending on the composite, size/shape and excitation) - much lower than the QYs of downconversion luminescence and not enough for really deep tissue imaging and therapy ${ }^{24,25}$. Although ex vivo study using $\mathrm{NaYbF}_{4}: \mathrm{Tm}^{3+} / \mathrm{CaF}_{2}$ of large amount and concentration demonstrated the imaging depth of $3.2 \mathrm{~cm}^{26,27}$, to date almost all the animal in vivo studies are still in the stage of the subcutaneous tumor model, where the involved imaging/therapy depth were actually not deep (1-cm level). To highlight the advantages and improve the applicability of UCNPs, it is very important to significantly increase the depth of imaging and therapy.

The absence of photobleaching and photoblinking, as well as the uniqueness of high spatial resolution, enable precise tracking of one single UCNP4. Thus, individual UCNPs possess such ideal properties suitable for single nanoparticles imaging/tracking, which enables one to use one single bright/small UCNP for probing a single protein, such as mapping a single protein moving through a cell, neuron cell interaction, and the process of brain cells connecting together to form a synapse 28,29 . However, most of UCNP-based microscopy studies were performed in laser scanning (confocal) modality, where a long scanning time is required ${ }^{19,20}$. It is because of the long lifetime (us-ms) and low QY of nanoparticles (statistically each ion only emits one photon during one lifetime period) that the dwelling time at each pixel is relatively long, disenabling rapid track events in the bioprocesses. Wide field microscopy with epi-illumination could be an alternative way to overcome this time-consuming scanning problem ${ }^{5}, 19$. However, larger excitation power (to reach a certain power density) would be necessary. In addition, it has to go through cell samples and growth media. This will induce a heating effect (which could damage cell activity $)^{21}$. More importantly, the unwanted scattering of both excitation and emission light will blur the image and reduce the resolution/contrast ${ }^{30}$.

The commonly used UCNPs employ $\mathrm{Yb}^{3+}$ ions as sensitizers, which have a strong absorption band at $974 \mathrm{~nm}$. However, this absorption band coincides with that of water, which causes light attenuation and heating. Due to the previous report it is effective to shift the excitation wavelength to the $915 \mathrm{~nm}$ absorption band of $\mathrm{Yb}^{3+}$ to improve the penetration depth and reduce the heating of tissue in imaging applications. Although this absorption band is weaker than the $975 \mathrm{~nm}$ band, excitation at this wavelength has been demonstrated to be very feasible and motivated for in vitro cell imaging, and imaging of very deeply embedded targets within tissues ${ }^{21,31-33}$. However, the condition determining which excitation wavelength for optimal use is not trivial and is related to the optical properties of the studied tissue and the imaging depth of interest. Thus consideration must be taken based on the experimental conditions. The impact of the optical properties of the tissue on the deep imaging ability of the $920 \mathrm{~nm}$ band is not clear yet. A further comprehensive study for tissues with different properties is also very important.

Similar to two-photon fluorescence from quantum dots and organic dyes, the QY of UCNPs is also power density dependent rather than a constant ${ }^{18}$. In this regard, one can use a larger power density (fluence rate inside the tissue) of a pulsed laser to give a "higher QY" of UCNPs before they get saturated. However, the saturation power density of UCNPs is not as large as those of organics dyes ${ }^{18}$. Another difference from the two-photon fluorescence microscopy is that the excitation of UCNPs relies on intermediate states of long lifetimes, which means that pulses shorter than a few milliseconds do not improve the QY substantially. Consequently, a microsecond or millisecond pulse laser with a high power density and acceptable average power would be a good choice for exciting UCNPs efficiently. Pulsed laser excitation of UCNPs has already been introduced in some reports, however, it was used just to perform lifetime measurement or control the emission spectrum ${ }^{34-36}$. How to improve the QY using a pulsed laser has not yet been studied nor mentioned.

In short, successful translations of the use of UCNPs from laboratories to clinics require further studies involving the exploitation of larger detection depth, higher QY and quicker microscopy. In this article we propose a novel evanescent wave (EW) excitation modality for rapid imaging and tracking of UCNPs as well as in vitro cancer cell membrane imaging. Experimental results show that this scanning-free method has advantages of rapid track, ultrahigh contrast and high spatiotemporal resolution. The impact of the tissue's optical properties on the deep imaging ability of a shifted excitation wavelength for fluorescence diffusion optical imaging (FDOI) is then investigated. All simulations with 
various tissue property parameters verify the deep imaging ability of $920 \mathrm{~nm}$ excitation. In our experiments a semiconductor $920 \mathrm{~nm}$ laser was used to excite UCNP inclusions in a tissue phantom at five depths. It was shown both experimentally and computationally that $920 \mathrm{~nm}$ laser excitation in UCNP-based FDOI could lead to a larger imaging depth range compared to that of traditional $974 \mathrm{~nm}$ excitation. Finally, a pulsed laser is proposed to replace a CW laser in order to improve the fluence rate within the tissue. With a doubled fluence rate, the quantum yield was also doubled.

\section{Nanoparticles Synthesis and Characteri- zation}

\section{Materials}

Lanthanide oxides $\left(\mathrm{Tm}_{2} \mathrm{O}_{3}, \mathrm{Yb}_{2} \mathrm{O}_{3}, \mathrm{Y}_{2} \mathrm{O}_{3}, \mathrm{Ho}_{2} \mathrm{O}_{3}\right.$ and $\left.\mathrm{Er}_{2} \mathrm{O}_{3}\right)$, trifluoroacetic acid $(99 \%)$, sodium trifluoroacetate $(98 \%)$, and 5-mercaptosuccinic acid (MSA) were purchased from Sigma-Aldrich. All lanthanide oxides utilized were $99.99 \%$ purity or higher. 1-Octadecene $(90 \%)$, oleic acid $(90 \%)$, anhydrous ethanol, anhydrous methanol, aqueous ammonia $(\sim 30 \%)$, cyclohexane and chloroform were purchased from Sinopharm Chemical Reagent Co. (China). Polyethylene glycol (PEG) (DSPE-mPEG-5000) was purchased from Creative PEGWorks, Inc. Intralipid was purchased from Zhejiang Univ. Hospital. The antibody anti-CEA8 (anti-CEAcam8/CD67) was purchased from Beijing Biosynthesis Biotechnology Co.,Ltd. HeLa cancer cell lines were supplied from the Medical School of Zhejiang University. All of the chemicals were used without further purification, and deionized (DI) water was used in the whole experimental procedure.

\section{Synthesis of $\mathrm{NaYF}_{4}: 20 \% \mathrm{Yb}^{3+} / 2 \% \mathrm{Er}^{3+}\left(\mathrm{Ho}^{3+}\right.$ or $\mathrm{Tm}^{3+}$ ) nanoparticles}

$\mathrm{NaYF}_{4}: 20 \% \mathrm{Yb}^{3+} / 2 \% \mathrm{Er}^{3+}\left(\mathrm{Ho}^{3+}\right.$ or $\left.\mathrm{Tm}^{3+}\right)$ were synthesized using a modified cothermalysis method ${ }^{32}$, 37. Herein we take the procedure of $\mathrm{NaYF}_{4}: \mathrm{Yb}^{3+} / \mathrm{Tm}^{3+}$ as an example and the other two were synthesized similarly. $0.025 \mathrm{mmol} \mathrm{Er}_{2} \mathrm{O}_{3}, 0.25 \mathrm{mmol} \mathrm{Yb}_{2} \mathrm{O}_{3}$ and $0.975 \mathrm{mmol} \mathrm{Y}_{2} \mathrm{O}_{3}$ were mixed and then solubilized in $20 \mathrm{~mL}$ of $50 \%$ aqueous trifluoroacetic acid at $80^{\circ} \mathrm{C}$ in a three-neck round-bottom flask. After about 2 hours the solution became clear and then the residual water and acid were slowly and completely evaporated at 85 ${ }^{\circ} \mathrm{C}$. Subsequently $5 \mathrm{~mL}$ DI water was added to re-dissolve the dried white powder. $4.5 \mathrm{mmol}$ of sodium trifluoroacetate was then added to the reaction vessel with $20 \mathrm{~mL}$ of 1 -octadecene $(90 \%)$ and $20 \mathrm{~mL}$ of oleic acid $(90 \%)$. The obtained solutions were slowly heated to $110{ }^{\circ} \mathrm{C}$ under vacuum with magnetic stirring for 40 minutes (to remove residual water and oxygen), during which the flask was purged periodically with dry argon gas. After the water was evaporated, the clear and yellow solution was heated to $310^{\circ} \mathrm{C}$ at a rate of about $20^{\circ} \mathrm{C}$ per minute under argon gas protection and kept at this temperature under vigorous stirring for about half an hour. Finally the heated solution was rapidly cooled down to $75^{\circ} \mathrm{C}$. $\mathrm{NaYF}_{4}: \mathrm{Yb}^{3+}$ $/ \mathrm{Er}^{3+}$ were precipitated by the addition of $50 \mathrm{~mL}$ ethanol and isolated via centrifugation at $3500 \mathrm{~g}$ for 5 mins. The resulting pellet was then washed twice with a mixture of methanol and ethanol (1:1) and isolated via centrifugation at $3500 \mathrm{~g}$ each time. The resulting muddy nanoparticles were dispersed in cyclohexane or chloroform by sonicating for 10 minutes for further characterization.

The optically equivalent phantom was prepared using intralipid and black ink with optical properties listed in Table 1 based on the report of time-of-flight measurement study ${ }^{38}$. The surface modification of UCNPs for in vitro cell membrane imaging was performed according to the previously reported method $^{21}$.

Table I. Optical properties for calculations (part) and experiments $\left(\mathrm{cm}^{-1}\right)$.

\begin{tabular}{llllll}
\hline$\lambda^{*}(\mathrm{~nm})$ & $\mu_{\mathrm{a}}$ (tissue) & $\mu_{\mathrm{s}}^{\prime}$ (tissue) & $\mu_{\mathrm{a}}$ (UCNPs) & $\begin{array}{l}\mu_{\mathrm{s}}{ }^{\prime} \\
(\mathrm{UCNPs})\end{array}$ & $\begin{array}{l}\eta \\
(\mathrm{UCNPs})\end{array}$ \\
\hline 974 & 0.54 & 5.5 & 0.06 & 1 & $0.3 \%$ \\
920 & 0.13 & 6.5 & 0.015 & 1 & $0.3 \%$ \\
800 & 0.08 & 8.0 & 0 & 1 & $0.3 \%$ \\
\hline
\end{tabular}

* denotes excitation or emission light wavelength.

\section{Characterizations}

These as-prepared UCNPs could form a clear, transparent colloidal suspension both in chloroform and cyclohexane. The size and shape of UCNPs were characterized by transmission electron microscopy (TEM). The representative TEM image in figure 1(a) showed that these were well-dispersed nanoparticles with a mean diameter of about $20 \mathrm{~nm}$ and a relatively narrow size distribution, favorable nanoparticle morphology for bioimaging and biosensing. Three kinds of different rare earth ions doped with $\mathrm{NaYF}_{4}$ : $\mathrm{Yb}^{3+} / \mathrm{Er}^{3+}, \mathrm{Yb}^{3+} / \mathrm{Ho}^{3+}$ and $\mathrm{Yb}^{3+} / \mathrm{Tm}^{3+}$ were synthesized separately, to emit light with different colors (red and green, as shown in figure 1(b)). 


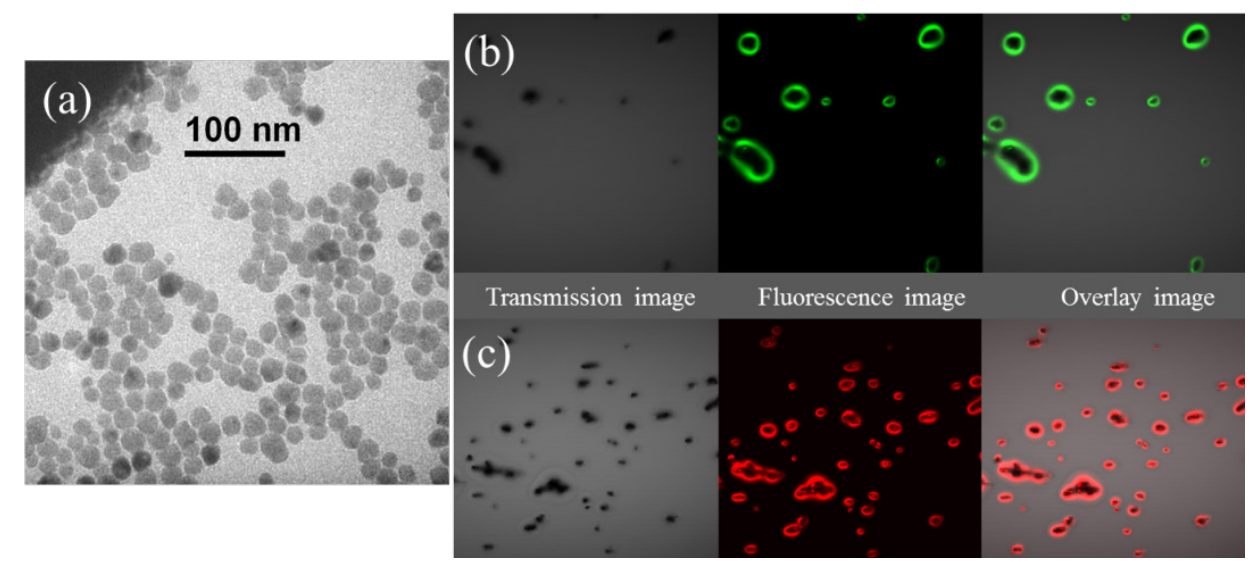

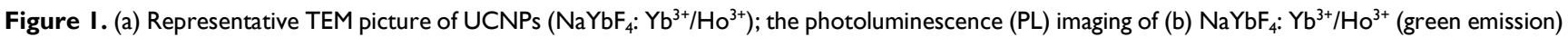
and (c) $\mathrm{NaYbF}_{4}: \mathrm{Yb}^{3+} / \mathrm{Er}^{3+}$ (red emission). The samples were dried on the glass slide before laser scanning microscopy experiments.

\section{Evanescent wave excitation for rapid mi- croscopy}

\section{System configuration and its working principle}

In total internal reflection microscopy (TIRM), a small portion of the reflected light penetrates through the interface and propagates parallel to the surface in the plane of incidence. This generates an electromagnetic field in the liquid adjacent to the interface. This field is termed the EW, and is capable of exciting emissive samples (or being scattered by nanoparticles) residing in the immediate region near the interface. The EW intensity I decays exponentially with the increasing distance $\mathrm{z}$ from the interface according to the following equation:

$$
\mathrm{I}(\mathrm{z})=\mathrm{I}_{0} \mathrm{e}^{-\mathrm{z} / \mathrm{d}}
$$

Here, $I_{0}$ is the intensity at the interface. The characteristic penetration depth $(d)$ at $\lambda$, the wavelength of incident light in a vacuum, is given by:

$$
d=\lambda /\left(4 \Pi\left(\left(n_{1} \sin \theta\right)^{2}-n_{2}^{2}\right)^{1 / 2}\right)
$$

where $n_{1}$ is the refractive index of the cover glass; $n_{2}$ is the refractive index of the water solution and $\theta$ is the incident angle on the glass/water interface. The penetration depth $d$ is independent of the polarization direction of the incident light, and decreases as the reflection angle gets larger. This value is also dependent upon the illumination wavelength and the refractive indices of the media present at the interface. In general, the value of $d$ is on the order of the incident wavelength, or perhaps somewhat smaller. When the incident angle equals the critical value, $d$ approaches infinity, and the wave-fronts of refracted light are normal to the surface.

In this work, the TIRM was performed based on an Olympus TIRM illuminator accessory for an inverted IX-71 microscope, as shown in Fig. 2 (a). A 980 $\mathrm{nm}$ single mode laser is coupled to the microscope via an external port. The alignment of the input fibre connector with the microscope optical path is adjustable in order to tune the incident light angle through a high NA objective. An adaptable Dichroic mirror and filters were used for excitation and emission light. The upconversion luminescence from UCNPs went into an EMCCD (Andor iXon3 885). Different from linear fluorescence, nonlinear power dependence $\left(I_{\mathrm{em}}\right.$ proportional to $\left.I_{\mathrm{ex}}{ }^{(n)}\right)$ of UCNPs will further shorten the detection depth. According to Eq. (2), the value of $d$ for $980 \mathrm{~nm}$ laser $\mathrm{EW}$ is $193.6 \mathrm{~nm}$ [ $\mathrm{n}_{1}$ (cover glass $)=1.518, \mathrm{n}_{2}$ (water) $=1.33$, incident angle $66^{\circ}$. The corresponding detection depths for two-photon excitation (green and red emission of UCNPs) and three-photon (blue and ultraviolet emission) excitation are $96.8 \mathrm{~nm}$ and $64.5 \mathrm{~nm}$, respectively. The three decay curves were shown in Fig. 1(b). Increasing the incident angle can enable smaller detection depth. The dimension of cell membrane thickness is on the order of $10 \mathrm{~nm}$. And thus EW excited UCNPs is capable of imaging a single events on the cell membrane with ultrahigh (both spectral and spatial) contrast ${ }^{39}$. Note that our proposed EW excitation modality for UCNPs can be used for both thin and thick samples because the PL detection could work in both backward and forward modes (prism-based TIRF), even though the imaging depth is smaller than $100 \mathrm{~nm}$. 

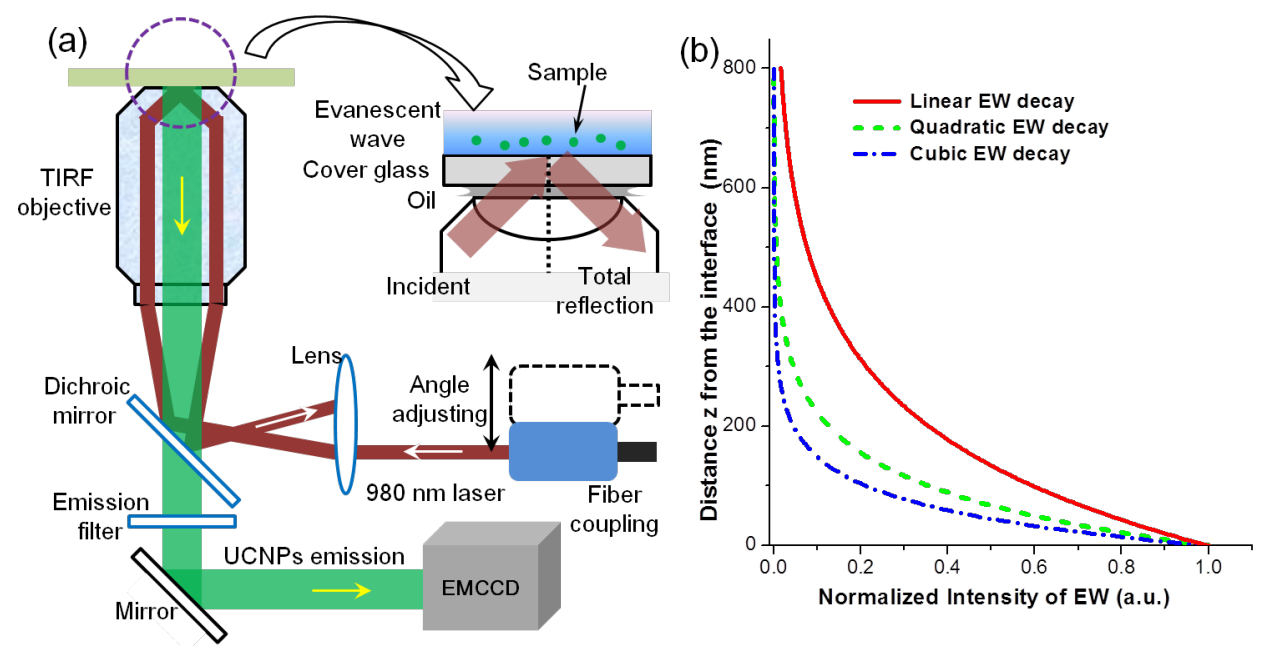

Figure 2. (a) Schematic diagram for the setup of the proposed objective-based TPEW imaging system. Detailed light-path is shown with the red indicating the excitation laser beam $(980 \mathrm{~nm})$ and the green light indicating the luminescence signal. TIRF objective: I00X, NA=I.45. (b) Calculated intensity decay curves of EW excited luminescence for three cases: linear excitation process, two-photon excitation process (green, red and NIR emission of UCNPs) and three-photon excitation process (blue emission of UCNPs).

\section{High speed particles tracking and cell mem- brane imaging}

The prepared UCNP samples were employed to perform fast nanoparticle tracking and live cell membrane imaging. TIRM imaging mode can only detect the UCNPs located within the depth of $96.8 \mathrm{~nm}$ (two-photon $\mathrm{NaYF}_{4}: \mathrm{Yb}^{3+} / \mathrm{Ho}^{3+}$ ) and thus eliminate the background of $\mathrm{z}$ direction. Our system can be easily switched between epifluorescence and through-the-objective TIRM illumination. The advantage of through-the-objective TIRM over epifluorescence was measured and analysed. Compared to Fig. 3(a), Fig. 3(b) has no scattering background signal, resulting in much higher signal noise ratio (SNR). In epi-fluorescence illumination, the excited sample region along the z-axis is relatively large (due to its poor $\mathrm{z}$-axis resolution) and excitation/emission light suffers from scattering (as demonstrated in Fig. 3(a)), which would blur the image. It's also worth pointing out that in a common wide field microscopy the excitation light would be strongly absorbed when going through the cell growth media (water solution), and consequently may induce photo-damage. This has been systematically studied in previous reports. On the contrary, TIRF has a very high axial resolution and no excitation energy to damage the sample. Furthermore, EW (evanescent wave) excited UCNPs are capable of imaging a single event/molecule of the cell membrane (important for the extended bioapplications), which cannot be implemented by wide field epi-fluoresence microscopy.
Then the highly diluted UCNP aqueous suspension of different emissions (only the result of $\mathrm{NaYF}_{4}: \mathrm{Yb}^{3+} / \mathrm{Er}^{3+}$ was shown in Fig. 3(a)) was used to perform particle (single particles or a few particles) tracking. EW excitation does not require any scanning and enables rapid real-time monitoring of the movement of single nanoparticles. The imaging stack was analysed with a $100 \mathrm{~ms}$ record time interval and the result of one particle was shown in pictures No. 1 to 25. The detailed moving trace of this UCNP can be recorded during the time of $2.5 \mathrm{~s}$, which probably is needed for finishing single image in the UCNP-based laser imaging system. Combining the benefit of TIRM and the ultrahigh sensitivity of EMCCD, ultra-fast imaging can be implemented in this system. As shown in Fig. 4 (b), in a large area (about 70um by 70 um) the image was captured at a rate of $350 \mathrm{fps}$ to track the trajectory of nanoparticle. The trajectory data were analysed and displayed in difference colours. A few particles imaging and fast tracking were well demonstrated to be significantly advantageous over the other UCNP imaging modality. The TIRM modality is a powerful tool for cell membrane study, which is very important for studying the interaction of UCNP and cell. In this work, surface-functionalized UCNPs were also employed to label HeLa cancer cells. The cancer cell experiments were performed similar to the previous work. As shown in Fig. 5, the positions of UCNPs luminescence agree well with the position of cancer cells, which demonstrate the successful live cell membrane imaging of MPEW excited UCNPs. 

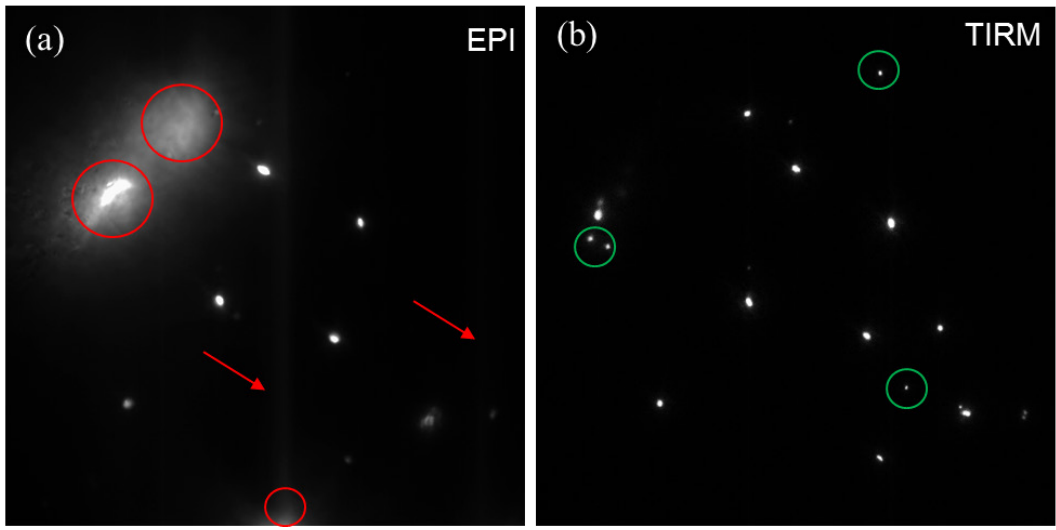

Figure 3. Comparison of luminescent microscopy from $\mathrm{NaYF}_{4}: \mathrm{Yb}^{3+} / \mathrm{Er}^{3+}$ in (a) epi-fluorescence imaging mode (red-indicated light was scattering/background) and (b) TIRM imaging mode at water-glass interfaces (green-indicated signal could be seen clearly).
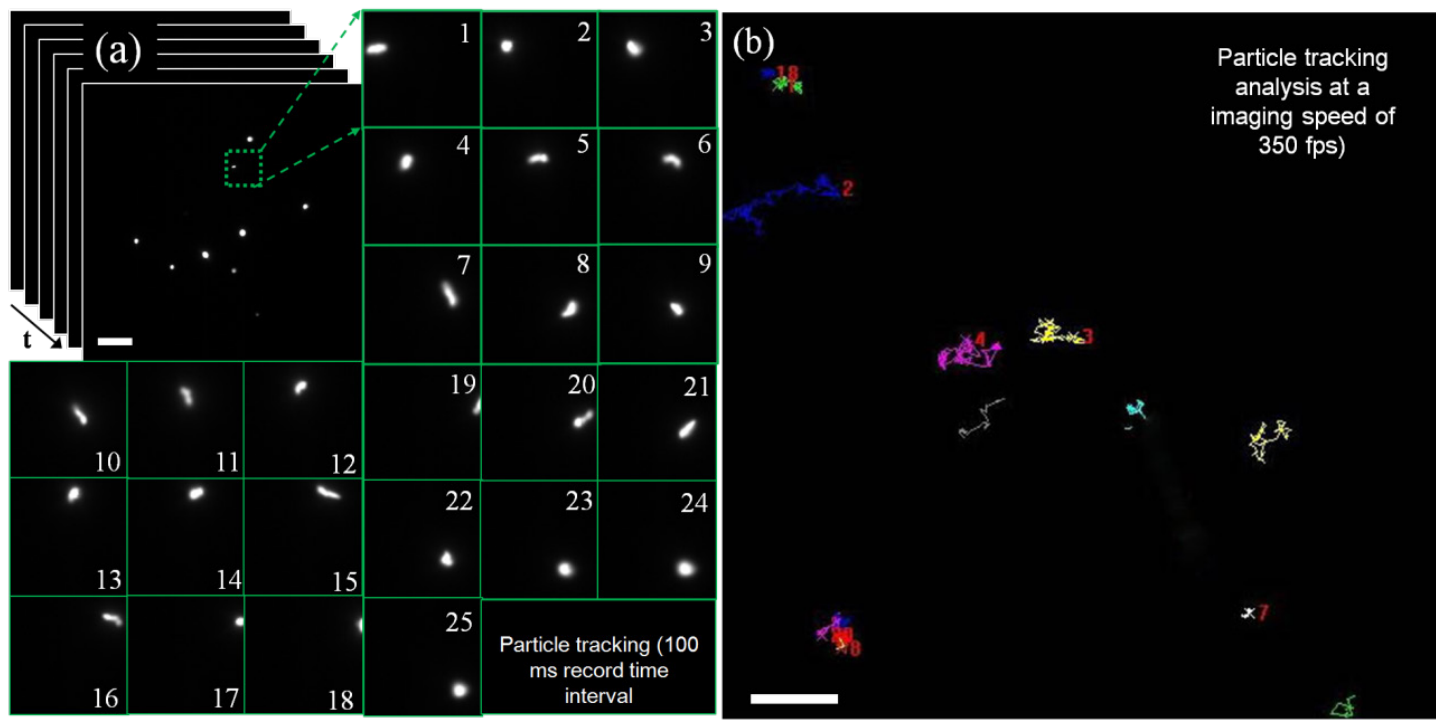

Figure 4. Rapid particle tracking in water solution (a) pictures No. I to 25 show the fast moving with a record time interval of $100 \mathrm{~ms}$; (b) tracks of nanoparticles in water solution with a very high imaging rate of $350 \mathrm{fps}$ (frame/second). The white scale bars are $10 \mathrm{um}$.
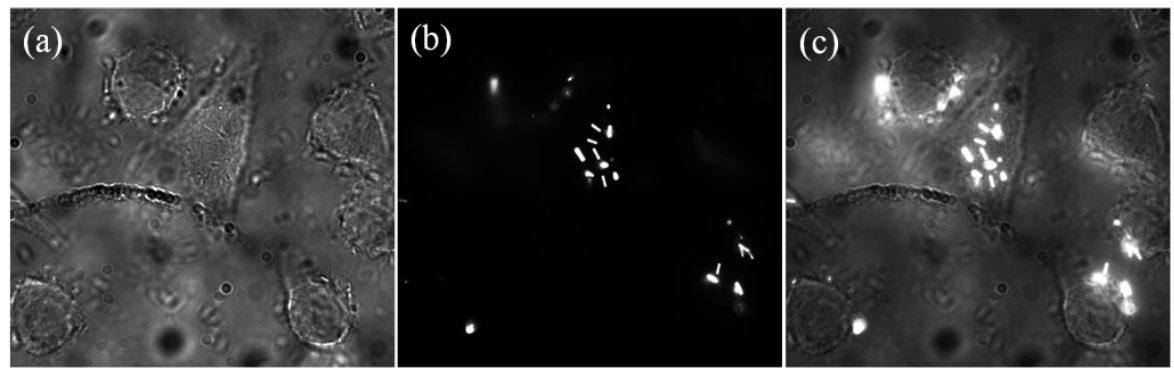

Figure 5. $\mathrm{NaYF}_{4}: 20 \% \mathrm{Yb}^{3+} / 2 \% \mathrm{Er}^{3+}$ assisted live cell membrane imaging with (a) bright field image, (b) $\mathrm{EW}$ excited luminescence image and (c) overlay image.

\section{Tissue property dependent imaging depth at $920-n m$ band}

The diffusion approximation for light propagation in biological turbid media can be modeled with diffusion equation $(D E)^{10}$. Fluorescence diffusing imaging in turbid media can be described as two DEs, with one describing the excitation light propagation and the other the fluorescence emission propagation. In the steady state, they can be written as

$$
\left(-D_{e x} \nabla^{2}+\mu_{\mathrm{a}}^{e x}\right) \Phi_{e x}(\mathbf{r})=S(\mathbf{r})
$$

and

$$
\left(-D_{e m} \nabla^{2}+\mu_{\mathrm{\alpha}}{ }^{\mathrm{em}}\right) \Phi_{\mathrm{em}}(\mathbf{r})=\eta(\mathbf{r}) \mu\left[\Phi_{e x}(\mathbf{r})\right]^{2}
$$

where $\mu_{\mathrm{a}}, \mu_{\mathrm{s}}{ }^{\prime}$, and $\mathrm{D}\left(=1 /\left(3 \mu_{\mathrm{a}}+3 \mu_{\mathrm{s}}{ }^{\prime}\right)\right.$ denote the absorp- 
tion, reduced scattering and diffusion coefficients of tissue (ex: excitation; em: emission), $\Phi(\mathbf{r})$ the photon fluence rate, $S(\mathbf{r})$ the source term, $\eta(\mathbf{r})$ the quantum yield of UCNPs, $\mu$ the absorption coefficient of UCNPs. Light in tissue is not only scattered but also absorbed. Due to the absorption, light intensity will be exponentially attenuated. Compared to $920 \mathrm{~nm}$ light, $974 \mathrm{~nm}$ light has much higher absorption in tissue. Therefore, $974 \mathrm{~nm}$ light would fade away much more quickly so that deep UCNP inclusion cannot be excited for emission. Excitation light propagation simulations in turbid media were made to compare the light fluence rate distributions of these two different excitation lasers (without UCNPs). The geometry and configurations for simulations were schematically given in Fig. 6(a). Typical optical parameters settings are listed in Table 1, calculated based on the reported method and data ${ }^{40,41}$. A circular laser beam (diameter: $10 \mathrm{~mm}$ ) illuminated the tissue from the center of the bottom. Picture (a) and (b) in Fig. 7 has shown the cross-sectional slices of $920 \mathrm{~nm}$ and $974 \mathrm{~nm}$ photon fluence rate $(\mathrm{u})$ distributions $(\log (\mathrm{u}))$, respectively. Due to much larger absorption coefficient, $974 \mathrm{~nm}$ light was rapidly absorbed by tissue while $920 \mathrm{~nm}$ laser light could penetrate through a deeper distance as shown in Fig. 2(a) and 2(b). Light fluence rate in Fig. 2(b) has a more concentrated distribution near the incident area.

Then equations (3) and (4) were used to calculate UC PL photon propagation with UCNP inclusion at five controlled depths $(2.5,6.5,10,13.5$, and 17.5 $(\mathrm{mm})$ ). The intensities of UC PL on the top surface boundary were exacted out and the ratio (920nm-excited over 974nm- excited) at each depth was calculated to compare their deep imaging ability. These ratio values were plotted as a function of depth, as shown in curve (2) in Fig. 2 (c) (Fig. 2 (d) as well). The intensity ratio was plotted as a function of inclusion depth (curve i in Fig. 2(c)). Since $974 \mathrm{~nm}$ light has a higher excitation efficiency the ratio value is less

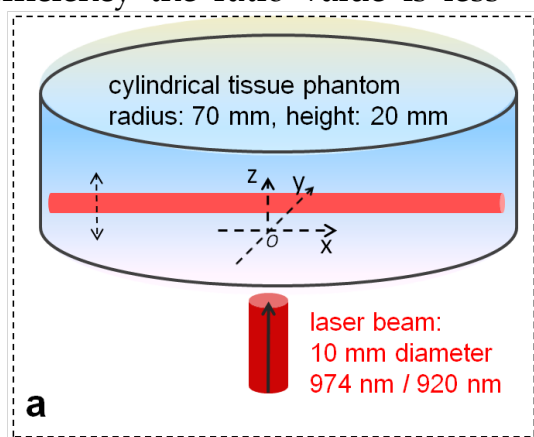

than 1 in the small depth range (about 0.063 at $2.5 \mathrm{~mm}$ depth). When it became deeper, more rapid attenuation of $974 \mathrm{~nm}$ light resulted in a sharp increase of this ratio. At the depth of $17.5 \mathrm{~mm}$ a ratio value of 2.9 was achieved, which means $920 \mathrm{~nm}$ light could excite nearly 3 times more UC photons than $974 \mathrm{~nm}$ light. Equivalent depth (ED) is defined as the UCNP depth of ratio value 1 . Theoretically the $\mathrm{ED}$ depends on the optical properties. Increased scattering and larger absorption ratio $(974 \mathrm{~nm} / 920 \mathrm{~nm})$ will both result in a smaller ED.

To investigate the impact of the optical properties of the tissue on the deep imaging ability of $920 \mathrm{~nm}$ excitation, four additional tissues with different absorption and scattering coefficients were studied in the calculation. With a fixed absorption coefficient (e.g., $0.54 \mathrm{~cm}^{-1} @ 974 \mathrm{~nm}$ ), two tissues were set with different reduced scattering coefficients $\left(e . g ., 4.5 \mathrm{~cm}^{-1}\right.$ and $\left.6.5 \mathrm{~cm}^{-1} @ 974 \mathrm{~nm}\right)$. The coefficients at other wavelengths were correspondingly changed using the calculation method and tissue components spectra in the reported work ${ }^{40,41}$. On the contrary, another group of tissues with different absorption coefficients (the reduced scattering is fixed) was also studied. Similarity, the ratio values $(920 \mathrm{~nm}$-excited over $974 \mathrm{~nm}$ - excited) at each depth were calculated for each case and the results were shown in Fig. 7(c). The $974 \mathrm{~nm}$ light absorption of tissue is dependent on the water contents of tissue species. Even though the deep imaging ability in tissue with lower absorption $\left(4.5 \mathrm{~cm}^{-1} @ 974\right.$ $\mathrm{nm})$ is not so remarkable as in the case of tissue with more water $\left(6.5 \mathrm{~cm}^{-1} @ 974 \mathrm{~nm}\right)$, a $920 \mathrm{~nm}$ laser is still advantageous over $974 \mathrm{~nm}$ when the imaging depth is larger than $1.4 \mathrm{~cm}$. In some tissues rich in water (e.g., prostate) the absorption coefficient is normally very large $\left(>6.5 \mathrm{~cm}^{-1}\right)$, and the ratio is up to 4 at the depth of $1.75 \mathrm{~cm}$. After comparisons of curves i, ii, iii and curves i, iv, v, the deep imaging ability of $920 \mathrm{~nm}$ excitation is significant in a wide range of tissue optical properties.

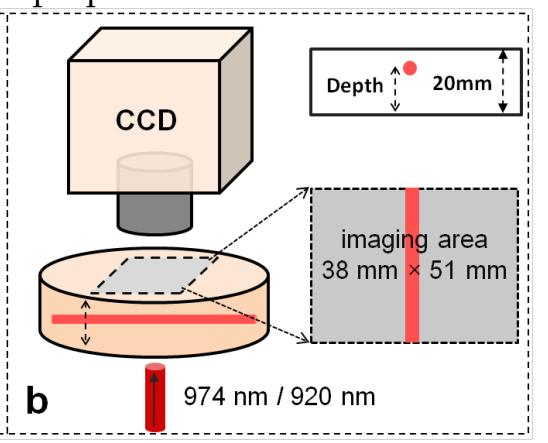

Figure 6. Schematics illustrating geometries and configurations of (a) simulation model and (b) FDOI experiments. A laser beam of circular shape illuminates on the bottom of the phantom. One CCD camera is used to capture an image for every depth and excitation on the other side. Depth of UCNP inclusions refers to vertical distance from tissue bottom to the center of glass tube. 


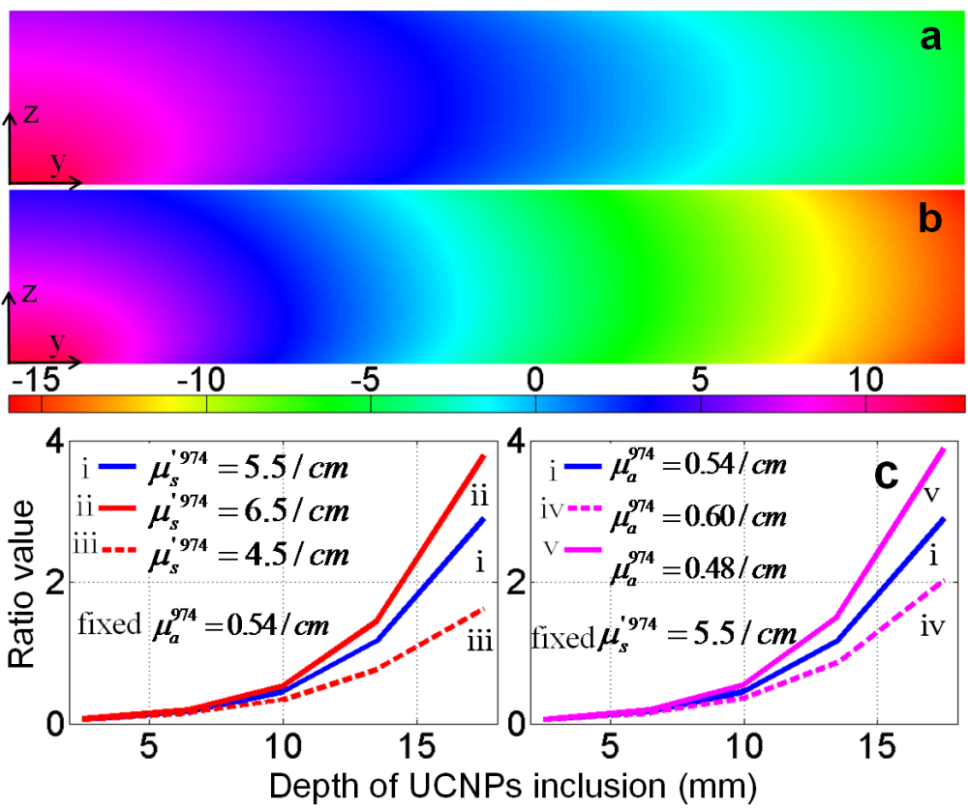

Figure 7. Cross-sectional slices of simulated (a) $920 \mathrm{~nm}$ and (b) $974 \mathrm{~nm}$ photon fluence rate (log(u)) distributions in yz half-plane; (c) ratio of UC PL at each depth (curve i ); ratio values for different scattering (curves ii and iii); ratio values for different absorption properties (curves iv and $v$ ); all the simulation results (curves ii - $\mathrm{v}$ in (c)) indicate that $920 \mathrm{~nm}$ light could excite UC PLs more efficiently in deep tissue with various optical tissue properties.

Then tissue phantom experiments were carried out to further validate our calculation studies. A glass tube filled with as-prepared clear $\mathrm{NaYF}_{4}: \mathrm{Yb}^{3+} / \mathrm{Tm}^{3+}$ chloroform suspension was embedded in the phantom at the previous five controlled depths. The phantom was imaged with the system depicted in Fig. 6(b). At each depth and each excitation the image was captured by CCD with the same exposure time (Fig. 8 (a) to 8 (j)). At the depth of $2.5 \mathrm{~mm}$, the emission from UCNP has to go through a long way of being scattered and absorbed before reaching the CCD camera, and consequently the outline of the tube at $2.5 \mathrm{~mm}$ depth cannot be seen while those at $15 \mathrm{~mm}$ and 17.5 $\mathrm{mm}$ can be seen clearly. The PL spectrum was analyzed with tunable liquid crystal filters. The peaks at $800 \mathrm{~nm}$ wavelength indicated that the PL was emitted from $\mathrm{NaYF}_{4}: \mathrm{Yb}^{3+} / \mathrm{Tm}^{3+}$. The two sets of normalized curves in the inset of Fig. $8(\mathrm{k})$ show the spectra and normalized intensities of UC PL excited by a $920 \mathrm{~nm}$ laser and $974 \mathrm{~nm}$ laser, respectively. With increased excitation depth, the boundary PL intensity diffusing from UCNP inclusions decrease because a deeper position requires the excitation light to experience much more scattering and absorption. After comparing these two sets of spectra one could easily see that the five peak intensities $\left(f^{\prime}-j^{\prime}\right)$ have a more rapid descent trend than those $\left(a^{\prime}-e^{\prime}\right)$ have, which is attributed to a much higher tissue absorption at $974 \mathrm{~nm}$. The calculated intensity ratio had a good agreement with the simulated results, as shown in curves of Fig. 8(k).

The factors affecting the QY of UCNPs include ion doping, host material, size and shape of nanoparticles, surface defects, external enhancement, etc. Although lots of efforts have been made to improve the emission efficiency of UCNPs, their QY is still relatively low. It is of utmost importance to substantially improve it to realize deep imaging and therapy. Due to the nonlinear dependence, its QY is also power density dependent. One commonly used excitation method is a CW laser, where larger excitation intensity for stronger UC PL may induce an overheating effect, because almost all the absorbed light energy will be transformed into heat, resulting in a significant local temperature rise. In principle, a pulsed laser with higher power density would increase the QY. The two-photon process of UCNPs relies on the long lifetime in the intermediate states rather than virtual states; the saturation power density is much lower than two photon organic dyes. Thus an ultrafast pulse laser (with pulse duration much shorter than lifetime of UCNPs) does not greatly improve the QY. A microsecond or millisecond pulsed laser could drastically improve the QY. Controlling the pulse width and repetition rate could generate an excitation of higher power density but with an acceptable average power, which is accessible using some commercially available laser. Alternatively, a cost effective way is to use a mechanical chopper to modulate a CW laser source, as shown in Fig. 9 (a). With a tunable modulation frequency, the dynamic ranges of the pulse width and repetition rate are very user-friendly. In this work, we demonstrated that a pulsed laser with 
higher power density could generate a higher fluence rate within the exposed tissue, which can improve the QY of UCNPs distributing within such a tissue. For simplicity, two laser sources with the same average power were set to $\mathrm{CW}$ and pulsed modes, as shown in Fig. 9 (b). The tissue sample was shown in Fig. 9 (c). In dimension, it is a cylinder tissue block with a radius of $3 \mathrm{~cm}$ and a length of $3 \mathrm{~cm}$, which is close to the case of small mouse experiments. The tissue optical properties were set to an absorption coefficient of $5.4 \mathrm{~cm}^{-1}$ and a reduced scattering coefficient of $5.5 \mathrm{~cm}^{-1}$. The wavelength of the laser is $974 \mathrm{~nm}$ and the average power is $500 \mathrm{~mW}$. The peak power and pulse width for the pulsed ( $5 \mathrm{KHz}$ modulation, square wave pulse) laser is $1 \mathrm{~W}$ and 200 us (comparable to the lifetime of UCNPs), respectively. Based on the diffusion equa- tion described in equation (3), the fluence rate within the tissue was calculated for both excitations. As shown in Fig. 10 (a), a higher power density pulsed laser induced a higher fluence rate inside the tissue. The QY of UCNPs is proportional to the excitation power density under unsaturated excitation. Based on the reported curve, the QY of UCNPs embedded within the tissue was also calculated for each depth, as shown in Fig. 10 (b). At each depth, a pulsed laser could generate a doubled QY compare to the CW excitation mode. Such doubled QY would definitely improve the deep imaging ability and PDT efficiency. This QY improvement only relies on the unique property of nonlinear PL process and has nothing to do with other factors, such as the absolute QY value, the size/shape of UCNPs, or the ions doping.

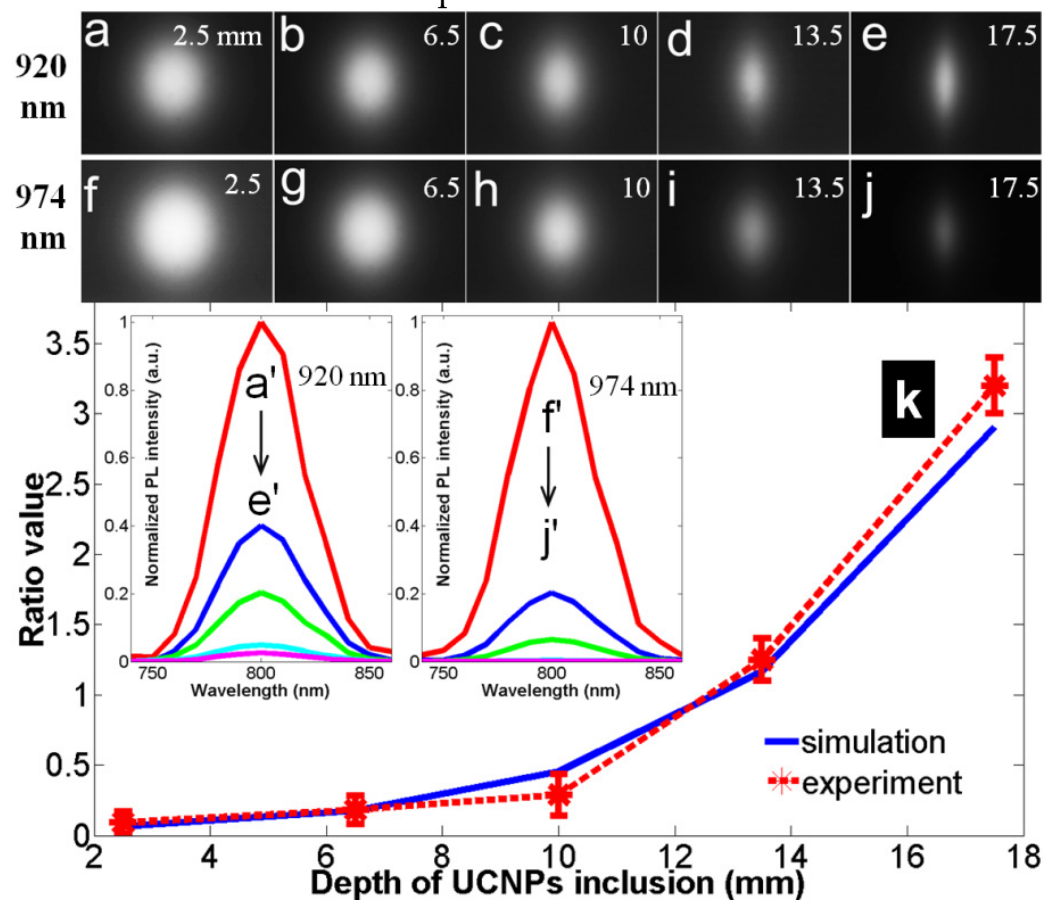

Figure 8. Experimental results: UC PL image $\left(38 \times 5 \mathrm{I} \mathrm{mm}{ }^{2}\right)$ at each depth and each excitation ( $920 \mathrm{~nm}$ excited: a-e, $980 \mathrm{~nm}$ excited: f-j); normalized UC PL spectra (the inset); ratio value at each depth compared to simulated results (the curves in k). Pulsed excitation to improve QY.

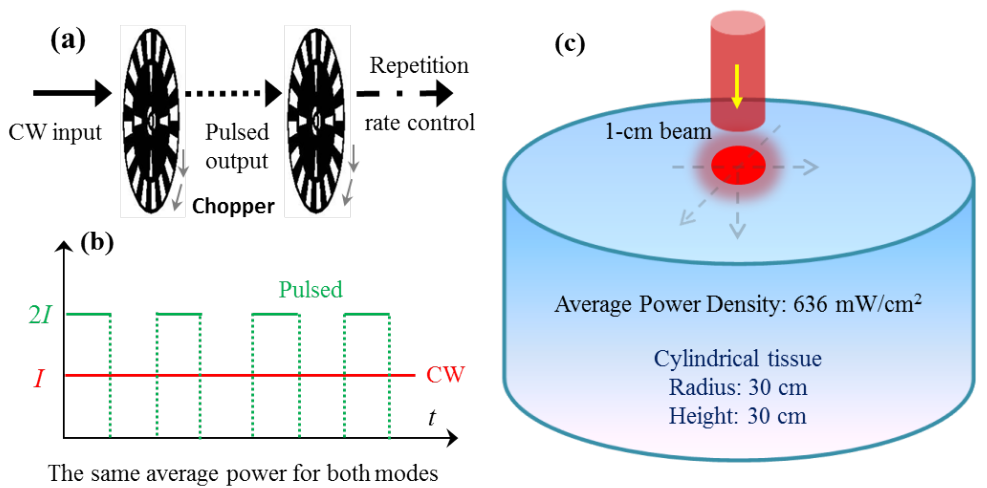

Figure 9. (a) The schematics of using chopper to modulate a $\mathrm{CW}$ laser and control the repetition rate, tunable modulation frequency $(\mathrm{Hz} \sim \mathrm{KHz})$ for the tunable width of pulse and repetition rate; (b) the case for calculation, two excitation lasers (CW and pulsed laser) with the same average power; (c) the schematics of tissue configurations for simulating photon fluence rate distributions and quantum yields within tissue. 

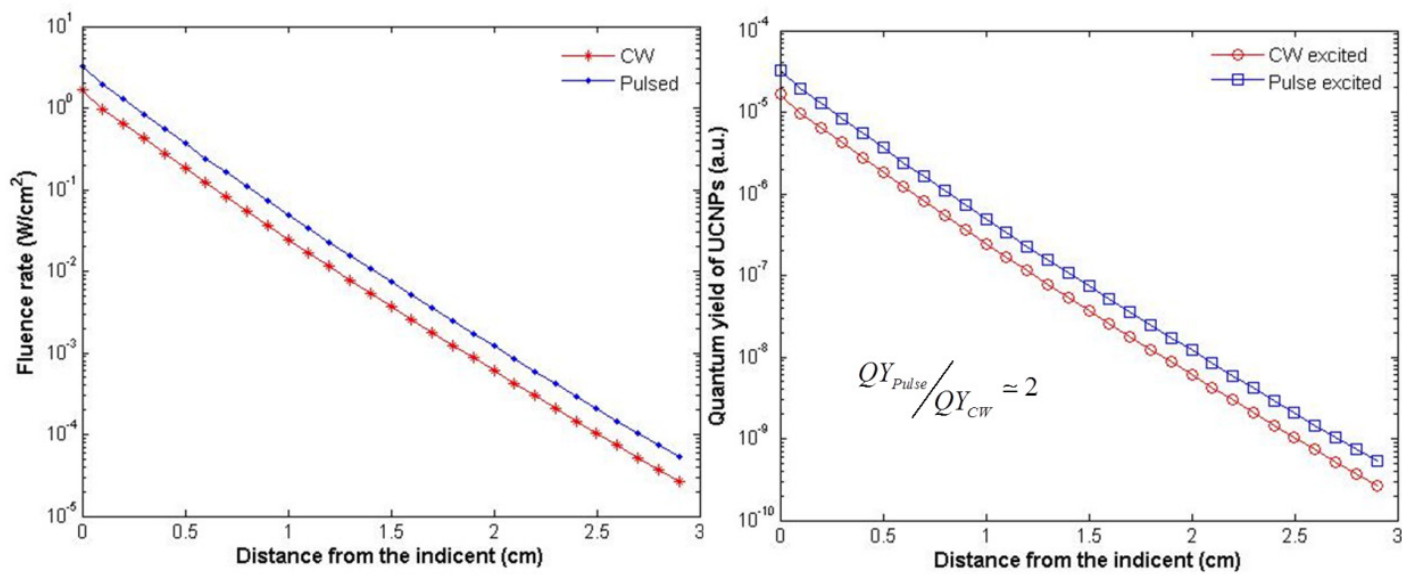

Figure 10. (a) Calculated fluence rates within tissue (with optical properties given in the text) under excitation by CW and pulsed $974 \mathrm{~nm}$, respectively; (b) the corresponding QYs for the UCNPs (excited by CW and pulsed laser) as a function of depth.

\section{Conclusion}

For the first time the EW has been proposed to excite lanthanide-doped UCNPs for microscopy. The analyzed experimental results - particles tracking and in vitro cell membrane imaging - have revealed that this new imaging method for UCNPs is non-scanning, ultrahigh contrast and high spatiotemporal resolution. The present novel technique will be a very powerful tool for many UCNP-based applications, such as single particles/molecules imaging, single proteins tracking and single events monitoring in the bioprocess. Studies with different tissues have been made to illustrate the impact of optical properties of the tissue on the deep imaging ability of $920 \mathrm{~nm}$ band excitation. With simulated and experimental results, the $920 \mathrm{~nm}$ laser has been shown to have deep imaging ability in a wide dynamic range of tissue optical properties. This study has substantially testified that a shifted wavelength - to avoid the strong light absorption of tissue - has remarkable feasibility and applicability. With the benefit of a power density dependent QY, a pulsed laser has been proposed to replace a $\mathrm{CW}$ laser to increase the QY of UCNPs. Our simulations clearly indicate that UCNPs embedded in the tissue can obtain a double QY under chopper modulated excitation. This proposal enables one to use a more effective pulse laser to further improve the imaging depth and PDT efficiency. In short, several optimizations of optical excitations of UCNPs have been comprehensively demonstrated for rapid microscopy, deeper tissue imaging and higher QY. These three methods/aspects about UCNP performance are related to each other. For example, pulsed excitation could be used in a TIRF method to further improve the speed of imaging, and appropriate excitation wavelength shift together with pulsed excitation could further improve the deep imaging ability. These proposed optical optimizations will improve the applicability of UCNPs and further catalyze their development in the field of biomedical optics.

\section{Abbreviations}

QY: quantum yield; UCNPs: upconversion nanoparticles; EW: evanescent wave; QDs: quantum dots; PDT: photodynamic therapy; EW: evanescent wave; FDOI: fluorescence diffusion optical imaging; MSA: 5-mercaptosuccinic acid; PEG: Polyethylene glycol; TEM: transmission electron microscopy; TIRM: total internal reflection microscopy; DE: diffusion equation; ED: equivalent depth.

\section{Acknowledgments}

This work was supported by Guangdong Innovative Research Team Program (No. 201001D0104799318), the National Natural Science Foundation of China (No. 91233208 and No. 61178062) and the Young Faculty Academic Training Foundation of SCNU (No. 2012KJ017).

\section{Competing Interests}

The authors have declared that no competing interest exists.

\section{References}

1. Xu CT, Zhan Q, Liu H, et al. Upconverting nanoparticles for pre-clinical diffuse optical imaging, microscopy and sensing: Current trends and future challenges. Laser \& Photonics Reviews. 2013;:1-35.

2. Xu CT, Svensson N, Axelsson J, et al. Autofluorescence insensitive imaging using upconverting nanocrystals in scattering media. Applied Physics Letters. 2008; 93(17):171103.

3. Zhou J, Liu Z, Li F. Upconversion nanophosphors for small-animal imaging. Chemical Society Reviews. 2012; 41(3):1323-1349.

4. Wu SW, Han G, Millirona DJ, et al. Non-blinking and photostable upconverted luminescence from single lanthanide-doped nanocrystals. 
Proceedings of the National Academy of Sciences of the United States of America. 2009; 106(27):10917-10921.

5. Nyk M, Kumar R, Ohulchanskyy TY, et al. High Contrast in Vitro and in Vivo Photoluminescence Bioimaging Using Near Infrared to Near Infrared Up-Conversion in $\mathrm{TM} 3+$ and $\mathrm{Yb} 3+$ Doped Fluoride Nanophosphors. Nano Letters. 2008; 8(11):3834-3838.

6. Yang $Y$, Shao $Q$, Deng $R$, et al. In Vitro and In Vivo Uncaging and Bioluminescence Imaging by Using Photocaged Upconversion Nanoparticles. Angewandte Chemie International Edition. 2012; 51(13):3125-3129.

7. Zhao L, Kutikov A, Shen J, et al. Stem Cell Labeling using Polyethylenimine Conjugated (a-NaYbF4:Tm3+)/CaF2 Upconversion Nanoparticles. Theranostics. 2013; 3(4):249-257.

8. Xiong LQ, Yang TS, Yang Y, et al. Long-term in vivo biodistribution imaging and toxicity of polyacrylic acid-coated upconversion nanophosphors. Biomaterials. 2010; 31(27):7078-7085.

9. Dong B, Cao B, He Y, et al. Temperature Sensing and In Vivo Imaging by Molybdenum Sensitized Visible Upconversion Luminescence of Rare-Earth Oxides. Advanced Materials. 2012; 24(15):1987-1993.

10. Xu CT, Axelsson J, Andersson-Engels S. Fluorescence diffuse optical tomography using upconverting nanoparticles. Applied Physics Letters. 2009; 94:251107.

11. Liu Q, Sun $\mathrm{Y}$, Li C, et al. 18F-Labeled Magnetic-Upconversion Nanophosphors via Rare-Earth Cation-Assisted Ligand Assembly. ACS Nano. 2011; 5(4): 3146-3157.

12. Zhou J, Yu MX, Sun $Y$, et al. Fluorine-18-labeled Gd3+/Yb3+/Er3+ co-doped NaYF4 nanophosphors for multimodality PET/MR/UCL imaging. Biomaterials. 2011; 32(4):1148-1156.

13. Chen ZG, Chen HL, Hu H, et al. Versatile synthesis strategy for carboxylic acid-functionalized upconverting nanophosphors as biological labels. Journal of the American Chemical Society. 2008; 130(10):3023-3029.

14. van de Rijke F, Zijlmans $\mathrm{H}$, Li S, et al. Up-converting phosphor reporters for nucleic acid microarrays. Nat Biotech. 2001; 19(3):273-276.

15. Qian HS, Guo HC, Ho PC-L, et al. Mesoporous-Silica-Coated Up-Conversion Fluorescent Nanoparticles for Photodynamic Therapy. Small. 2009; 5(20):2285-2290.

16. Idris NM, Gnanasammandhan MK, Zhang J, et al. In vivo photodynamic therapy using upconversion nanoparticles as remote-controlled nanotransducers. Nature Medicine. 2012; 18(10):1580-1585.

17. Boyer JC, van Veggel FCJM. Absolute quantum yield measurements of colloidal NaYF4: Er3+, Yb3+ upconverting nanoparticles. Nanoscale. 2010; 2:1417-1419.

18. Xu CT, Svenmarker P, Liu H, et al. High-Resolution Fluorescence Diffuse Optical Tomography Developed with Nonlinear Upconverting Nanoparticles. ACS Nano. 2012; 6(6):4788-4795.

19. Pichaandi J, Boyer J-C, Delaney KR, et al. Two-Photon Upconversion Laser (Scanning and Wide-Field) Microscopy Using Ln3+-Doped NaYF4 Upconverting Nanocrystals: A Critical Evaluation of their Performance and Potential in Bioimaging. The Journal of Physical Chemistry C. 2011; 115(39):19054-19064.

20. Gainer CF, Utzinger U, Romanowski M. Scanning two-photon microscopy with upconverting lanthanide nanoparticles via Richardson-Lucy deconvolution. Journal of Biomedical Optics. 2012; 17(7):076003-076001.

21. Zhan $\mathrm{Q}$, Qian $\mathrm{J}$, Liang $\mathrm{H}$, et al. Using $915 \mathrm{~nm}$ Laser Excited Tm3+/Er3+/Ho3+-Doped NaYbF4 Upconversion Nanoparticles for in Vitro and Deeper in Vivo Bioimaging without Overheating Irradiation. ACS Nano. 2011; 5(5):3744-3757.

22. Kangasniemi M, McNichols RJ, Bankson JA, et al. Thermal therapy of canine cerebral tumors using a $980 \mathrm{~nm}$ diode laser with MR temperature-sensitive imaging feedback. Lasers in Surgery and Medicine. 2004; 35(1):41-50.

23. Tabakoglu HO, Topaloglu N, Gulsoy M. The Effect of Irradiance Level in 980-nm Diode Laser Skin Welding. Photomedicine and Laser Surgery. 2010; 28(4):453-458

24. Chen G, Ohulchanskyy TY, Liu S, et al. Core/Shell NaGdF4:Nd3+/NaGdF4 Nanocrystals with Efficient Near-Infrared to Near-Infrared Downconversion Photoluminescence for Bioimaging Applications. ACS Nano. 2012; 6(4):2969-2977.

25. Chen G, Ohulchanskyy TY, Kachynski A, et al. Intense Visible and Near-Infrared Upconversion Photoluminescence in Colloidal LiYF4:Er3+ Nanocrystals under Excitation at $1490 \mathrm{~nm}$. ACS Nano. 2011; 5(6):4981-4986

26. Chen G, Shen J, Ohulchanskyy TY, et al. (a-NaYbF4:Tm3+)/CaF2 Core/Shell Nanoparticles with Efficient Near-Infrared to Near-Infrared
Upconversion for High-Contrast Deep Tissue Bioimaging. ACS Nano. 2012; 6(9):8280-8287.

27. Chen GY, Yang CH, Prasad PN. Nanophotonics and Nanochemistry: Controlling the Excitation Dynamics for Frequency $\mathrm{Up}$ - and Down-Conversion in Lanthanide-Doped Nanoparticles. Accounts of Chemical Research. 2013; [Epub ahead of print].

28. Ostrowski AD, Chan EM, Gargas DJ, et al. Controlled Synthesis and Single-Particle Imaging of Bright, Sub-10 nm Lanthanide-Doped Upconverting Nanocrystals. ACS Nano. 2012; 6(3):2686-2692.

29. Qiu $H$, Chen $G$, Sun $L$, et al. Ethylenediaminetetraacetic acid (EDTA)-controlled synthesis of multicolor lanthanide doped BaYF5 upconversion nanocrystals. Journal of Materials Chemistry. 2011; 21(43):17202-17208.

30. Paige MF, Bjerneld EJ, Moerner WE. A Comparison of Through-the-Objective Total Internal Reflection Microscopy and Epifluorescence Microscopy for Single-Molecule Fluorescence Imaging. Single Molecules. 2001; 2(3):191-201.

31. Vetrone F, Naccache R, Zamarrón A, et al. Temperature Sensing Using Fluorescent Nanothermometers. ACS Nano. 2010; 4(6):3254-3258.

32. Chen GY, Ohulchanskyy TY, Kumar R, et al. Ultrasmall Monodisperse NaYF4:Yb3+/Tm3+ Nanocrystals with Enhanced Near-Infrared to Near-Infrared Upconversion Photoluminescence. ACS Nano. 2010; 4(6):3163-3168.

33. Zou WQ, Visser C, Maduro JA, et al. Broadband dye-sensitized upconversion of near-infrared light. Nature Photonics. 2012; 6:560-564.

34. Schietinger $\mathrm{S}$, Aichele $\mathrm{T}$, Wang $\mathrm{HQ}$, et al. Plasmon-Enhanced Upconversion in Single NaYF4:Yb3+/Er3+ Codoped Nanocrystals. Nano Letters. 2009; 10(1):134-138.

35. Christian FG, Joshua GS, Channa RD, et al. Control of green and red upconversion in NaYF4:Yb3+,Er3+ nanoparticles by excitation modulation. J Mater Chem. 2011; 21:18530.

36. Zhang W, Ding F, Chou SY. Large Enhancement of Upconversion Luminescence of NaYF4:Yb3+/Er3+ Nanocrystal by 3D Plasmonic Nano-Antennas. Advanced Materials. 2012; 24(35):OP236-OP241.

37. Boyer JC, Cuccia LA, Capobianco JA. Synthesis of colloidal upconverting NaYF4 : Er3+/Yb3+ and Tm3+/Yb3+ monodisperse nanocrystals. Nano Letters. 2007; 7(3):847-852.

38. Alerstam E, Andersson-Engels S, Svensson T. White Monte Carlo for time-resolved photon migration. J Biomed Opt. 2008; 13(4): 041304.

39. Jaiswal JK, Simon SM. Imaging single events at the cell membrane. Nat Chem Biol. 2007; 3(2):92-98.

40. Alexandrakis G, Rannou FR, Chatziioannou AF. Tomographic bioluminescence imaging by use of a combined optical-PET (OPET) system: a computer simulation feasibility study. Physics in Medicine and Biology. 2005; 50(17):4225-4241

41. [Internet] Oregon Medical Laser Center of Oregon Health and Sciences University. http://omlc.ogi.edu/spectra/ 